\title{
Pattern Formation in Heterostructured Gel by the Ferrocyanide-Iodate-Sulfite Reaction
}

\author{
Tomonaga Ueno ${ }^{1 *}$, Ryo Yoshida ${ }^{2 *}$
}

1 Department of Chemical Systems Engineering, Graduate School of Engineering, Nagoya University

Furocho, Chikusa-ku, Nagoya 464-8603 (Japan),

ueno.tomonaga@material.nagoya-u.ac.jp

2 Department of Materials Engineering, School of Engineering, The University of Tokyo 7-3-1 Hongo, Bunkyo-ku, Tokyo 113-8656 (Japan), Fax: (+81)-3-5841-7112

ryo@cross.t.u-tokyo.ac.jp 
SI 1. The chemical states of the solution in reactor B

The chemical states of the solution in reactor $\mathrm{B}$ were measured with a fine $\mathrm{pH}$ electrode as shown in Figure S1. Other experimental conditions were the same as those found in section 2.2 of the main manuscript.

The chemical states depend on the flow rates as shown in Figure S2. The low-pH states were kept at a flow rate lower than $0.25 \mathrm{~mL} \mathrm{~min}^{-1}$ and the high-pH states were kept at flow rate higher than $0.6 \mathrm{~mL} \mathrm{~min}^{-1}$. A pH oscillating reaction occurs between the flow rates of $0.25 \mathrm{~mL}$ $\min ^{-1}$ and $0.6 \mathrm{~mL} \mathrm{~min}^{-1}$.

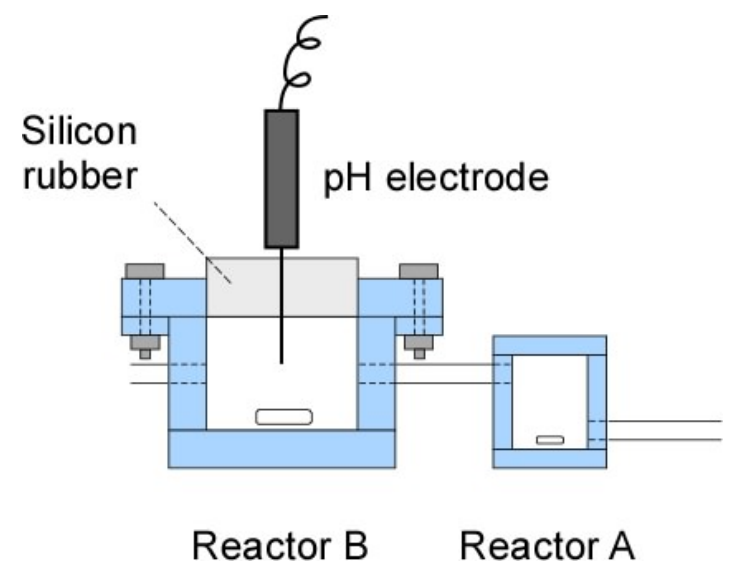

Figure S1. Set-up for the $\mathrm{pH}$ measurement of the solution in reactor B.

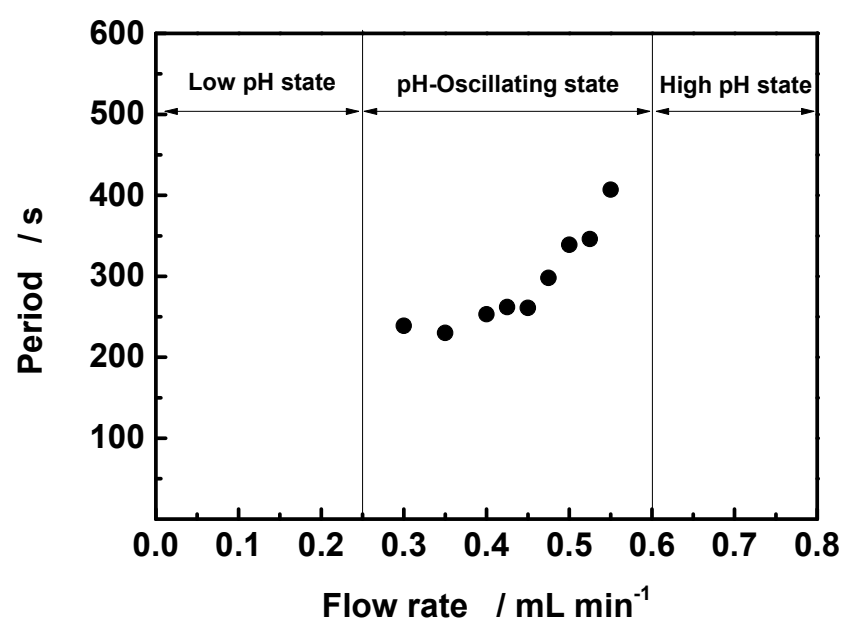

Figure S2. Chemical state diagram of reactor B at $50{ }^{\circ} \mathrm{C}$. 
SI 2. Pattern formation in a uniform gel

\section{SI 2.1 Material}

The acrylamide (Acros organics; 99\% for biochemistry, electrophoresis grade), cross-linker, $N, N$ '-methylenebisacryamide (Acros organics; 99\% for biochemistry for electrophoresis), accelerator, triethanolamine (Kanto Chemical. Co., Inc.), and initiator, ammonium persulfate (MP Biomedicals) were dissolved in distilled water, previously purged with nitrogen gas. The monomer concentration was $10 \mathrm{wt} \%$. The mole ratio of cross-linker to monomer was 3.6 to 96.4. The solution was injected into the space between two glass plates separated with $0.2 \mathrm{~mm}$ silicone rubber sheets. The gel was synthesized at $4{ }^{\circ} \mathrm{C}$ for $24 \mathrm{~h}$. The dialysis of the gel was conducted for 1 week.

\section{SI 2.2 Experimental condition for pattern formation}

The experimental condition was similar to that described in section 2.2 (Experiment for pattern formation) in the main manuscript.

\section{SI 2.3 Pattern formation in a uniform gel}

The pattern formations in the uniform polyacrylamide gel at respective flow rates are shown in Figure S3(a) and (b). At a flow rate of lower than $1.6 \mathrm{~mL} \mathrm{~min}^{-1}$, the chemical states were maintained at a low $\mathrm{pH}$ state. Alternately, over the flow rate of $2.1 \mathrm{~mL} \mathrm{~min}{ }^{-1}$, the chemical states were maintained at a high $\mathrm{pH}$ state. At a flow rate of $1.7 \mathrm{~mL} \mathrm{~min}^{-1}$, a self-replicating spot pattern appears, whereas at the flow rates of 1.8 and $1.9 \mathrm{~mL} \mathrm{~min}^{-1}$, a lamellar pattern appears. At a flow rate of $2.0 \mathrm{~mL} \mathrm{~min}{ }^{-1}$, an oscillating pattern is observed. The line profile along the yellow line of the lamellar pattern is shown in Figure S3(c). The periodicity of the low $\mathrm{pH}$ lines in the lamellar pattern is between 1.0 and $1.3 \mathrm{~mm}$. 
(a)

Low $\mathrm{pH}$ state

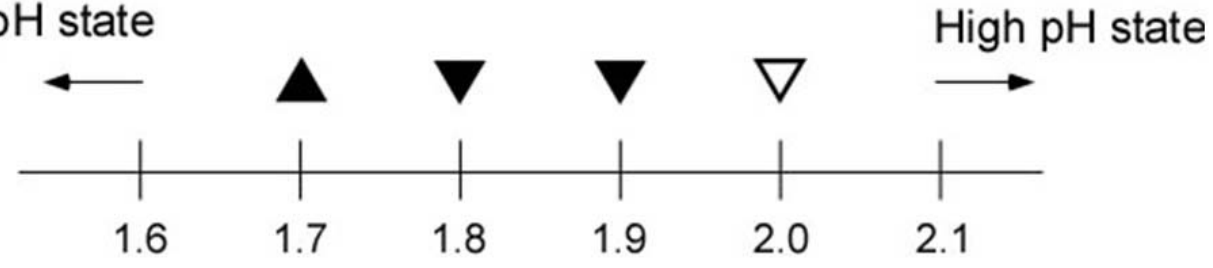

Flow rate $/ \mathrm{mL} \mathrm{min}^{-1}$

(b)

\section{$\boldsymbol{\Delta}$ : Self-replicating spots}

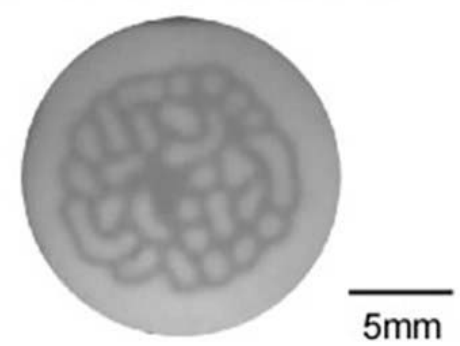

$1.7 \mathrm{~mL} \mathrm{~min}^{-1}$ $\overline{5 \mathrm{~mm}}$ $\boldsymbol{\nabla}$ : Lamellar

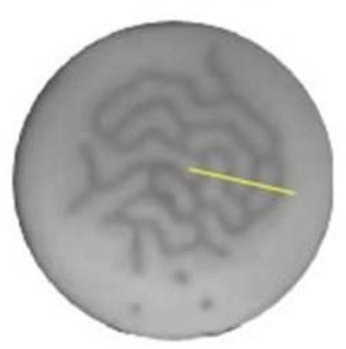

$1.9 \mathrm{~mL} \min ^{-1}$ (c)

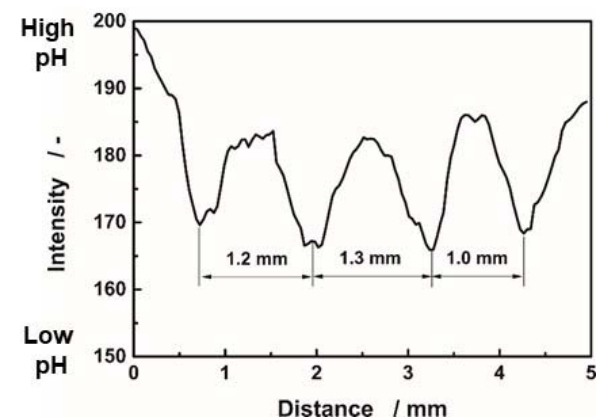

Figure S3. Pattern formation in a uniform gel at $50^{\circ} \mathrm{C}$. (a) Chemical states at respective flow rates. $\boldsymbol{\Delta}$ : Self-replicating spots; $\boldsymbol{\nabla}$ : lamellar patterns; $\nabla$ : Oscillating spots. (b) Selfreplicating and lamellar patterns, and (c) the line profile along the yellow line of the lamellar pattern.

\section{Supplementary Movie 1}

Description:

The pattern formation changes in the heterostructured gel when the flow rate was decreased at a constant flow rate of $0.34 \mathrm{~mL} \mathrm{~h}^{-1}$. The movie speed is $400 \times$ actual speed. 